\title{
COHN-LEAVITT PATH ALGEBRAS AND THE INVARIANT BASIS NUMBER PROPERTY
}

\author{
MÜGE KANUNI AND MURAD ÖZAYDIN
}

\section{INTRODUCTION}

Leavitt Path Algebras were defined just over a decade ago [1, 5], but they have roots in the works of Leavitt in the 60s focused on understanding the nature of the failure of the IBN (Invariant Basis Number) property for arbitrary rings [8].

A ring has IBN if any two bases of a finitely generated free module have the same number of elements. Fields, division rings, commutative rings, Noetherian rings all have IBN. A classical example of a ring without IBN is the algebra of endomorphisms of a countably infinite dimensional vector space. The free module of rank 1 over this ring has bases of $n$ elements for any positive integer $n$. In the early 60s William Leavitt asked and then answered this question: Given any positive integers $m<n$ is there a ring $R$ having a free module with a basis of $m$ elements and another basis with $n$ elements but no bases with $k$ elements if $k<n$ and not equal to $m$ ? Such a ring is said to be non-IBN of type $(m, n)$.

The algebras Leavitt constructed are denoted by $L(m, n)$ and nowadays called the Leavitt algebras. They have a semi-universal property: for any algebra $A$ satisfying $A^{m} \cong A^{n}$ as $A$-modules there is an algebra homomorphism (not necessarily unique) from $L(m, n)$ to $A$. They are simple if and only if $m=1,9$. The fact that a free $L(m, n)$ module of rank $m$ has a basis of $n$ elements is immediate from Leavitt's definition. However, to show that there are no bases of $k$ elements with $m<k<n$ requires an algebraic invariant, namely $\mathcal{V}(R)$ usually referred to as nonstable $\mathcal{K}$-theory.

$\mathcal{V}(R)$ is the monoid of isomorphism classes of finitely generated projective $R$-modules under direct sum. The Grothendieck group of $\mathcal{V}(R)$ is denoted by $\mathcal{K}_{0}(R)$. It turns out that $\mathcal{K}_{0}(R)$ (in fact, $\mathcal{K}_{0}(R) \otimes \mathbb{Q}$ ) suffices to determine whether $R$ has IBN or not ( [4, Corollary 5.7], also Proposition 10 below). However, $\mathcal{K}_{0}(R)$ cannot detect the type $(m, n)$ when $R$ is non-IBN. Another reason to work with $\mathcal{V}(R)$ rather than $\mathcal{K}_{0}(R)$ is to understand the $\mathcal{K}$-theory of corner subrings of $R$.

2010 Mathematics Subject Classification. Primary 16E20 Secondary 16S99.

Key words and phrases. Cohn-Leavitt path algebra, Invariant Basis Number, Morita Equivalence. 
For any idempotent $\alpha \in R, \alpha R \alpha$ is the corner ring associated with $\alpha$. While $\mathcal{V}(\alpha R \alpha)$ is (isomorphic to) a submonoid of $\mathcal{V}(R)$, the corresponding does not hold for the $\mathcal{K}_{0}$ groups (Theorem 15 and Example 19 below). In particular, $\mathcal{K}_{0}(R)$ cannot detect whether $\alpha R \alpha$ has IBN or not, but $\mathcal{V}(R)$ does, as well as is determining its type $(m, n)$ when it is non-IBN.

In general, it is difficult to compute $\mathcal{K}_{0}(R)$, even harder to compute $\mathcal{V}(R)$ for an arbitrary ring. However, in [6] George Bergman gave a construction for a ring $R$ whose $\mathcal{V}(R)$ is any given commutative finitely generated monoid satisfying two obviously necessary conditions. Leavitt path algebras were shown to fit the Bergman scheme in [5] and this was extended to Cohn-Leavitt path algebras of a separated di(rected )graph in [3]. Specifically, they showed that $\mathcal{V}(R)$ is isomorphic to the monoid of the (separated) digraph which is given explicitly with generators and relations [3, Theorem 4.3].

The main purpose of this note is to give an easily checked criterion to determine whether a separated Cohn-Leavitt path algebras has IBN (Theorem 13). An immediate consequence is that separated Cohn path algebras have IBN, which generalizes the main result in [2]. We also describe the non-stable $\mathcal{K}$-theory of a corner ring explicitly from the non-stable $\mathcal{K}$-theory of the ring (Theorem 15).

A proof of Theorem [13 in the special case of Leavitt path algebras was presented during the CIMPA 2015 Research School in Izmir by the first named author. The videos of all the lectures are publicly available at the website http://nesinkoyleri.org/eng/events/2015-lpa/. Below we give a more algebraic proof, but we also sketch the original proof.

In Section 2 below we give a geometric representation of a commutative semigroup defined by generators and relations. We also define graph semigroups, the Grothendieck group construction and a closure operator on a semigroup. We list some basic properties that we will use and give a few examples and counterexamples. Proposition 7 proves that the Grothendieck group of the semigroup of isomorphism classes of finitely generated free $L$ modules over a separated Cohn-Leavitt path algebra $L$ embeds in $\mathcal{K}_{0}(L)$.

Section 3 and 4 are the core of our paper. In Section 3, Theorem 13 gives the necessary and sufficient condition for a separated Cohn-Leavitt path algebra of a finite digraph to have IBN. As a consequence, we show that the separated Cohn path algebras have IBN (Corollary 14).

In Section 4, Theorem 15 gives the non-stable $\mathcal{K}$-theory of a corner ring explicitly in terms of the non-stable $\mathcal{K}$-theory of the ring. It proves that $\mathcal{V}(\alpha R \alpha)$ embeds in $\mathcal{V}(R)$ and also shows that $\mathcal{V}(\alpha R \alpha)$ is isomorphic to the closure of the semigroup generated by the isomorphism 
class of $\alpha R$ in $\mathcal{V}(R)$. Theorem 18 states a necessary condition for a corner algebra of a separated Cohn-Leavitt path algebra of a finite graph to have IBN. We also provide an example to show that this condition is not sufficient in general. In Example 20, we provide Morita equivalent rings which are non-IBN, but are of different types.

\section{Preliminaries}

2.1. Finitely Presented Commutative Semigroups. Let $\Omega$ be a finite set of generators and

$$
\left\{x_{i}=y_{i}: x_{i}, y_{i} \in \mathbb{N} \Omega \backslash\{\mathbf{0}\}\right\}_{i=1}^{n}
$$

be the relations. Consider the graph with vertex set $\mathbb{N} \Omega \backslash\{\mathbf{0}\}$. Each relation $x_{i}=y_{i}$, gives an edge $W_{i}$ joining $x_{i}$ to $y_{i}$. Let the edges of this graph be all translates of the $W_{i}$, $i=1, \ldots, n$ by elements of $\mathbb{N} \Omega \backslash\{\mathbf{0}\}$. The path components of this graph yield the geometric representation of the semigroup $S$ generated by $\Omega$ subject to these relations. (If the nodes are connected via any sequence of edges, then they are in the same equivalence class. Each path component corresponds to a distinct element of $S$.) The addition on $S$ descends from the addition of $\mathbb{N} \Omega \backslash\{\mathbf{0}\}$.

Example 1. Take $\Omega=\{v, w\}$, and the relations $R_{1}: v=2 w$ and $R_{2}: v=5 w$. To emphasize the difference we mark the relation and its translates of $R_{1}$ with blue and $R_{2}$ with red. Then, the geometric representation of $\mathbb{N} \Omega \backslash\{\mathbf{0}\}$ subject to $\left\{R_{1}, R_{2}\right\}$ is given by

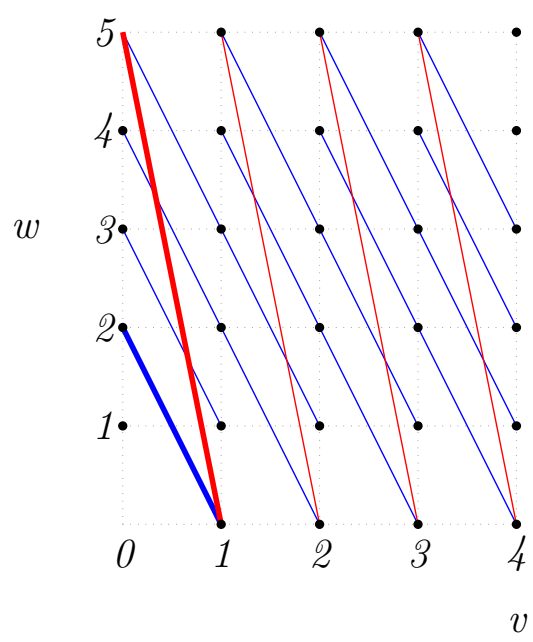

If we take $\mathbb{Z} \Omega$ as vertices and translates of the $W_{i}$ above by $\mathbb{Z} \Omega$ as edges, then the connected components give the Grothendieck group of the semigroup $S$ to be explained below. 
Example 2. Take $\Omega=\{v, w\}$, and the relation $R_{1}: v=v+w$. The geometric representation of $\mathbb{N} \Omega \backslash\{\mathbf{0}\}$ subject to $\left\{R_{1}\right\}$ is given by the figure on the left and $\mathbb{Z} \Omega /\left\langle R_{1}\right\rangle$ is given by the figure on the right.
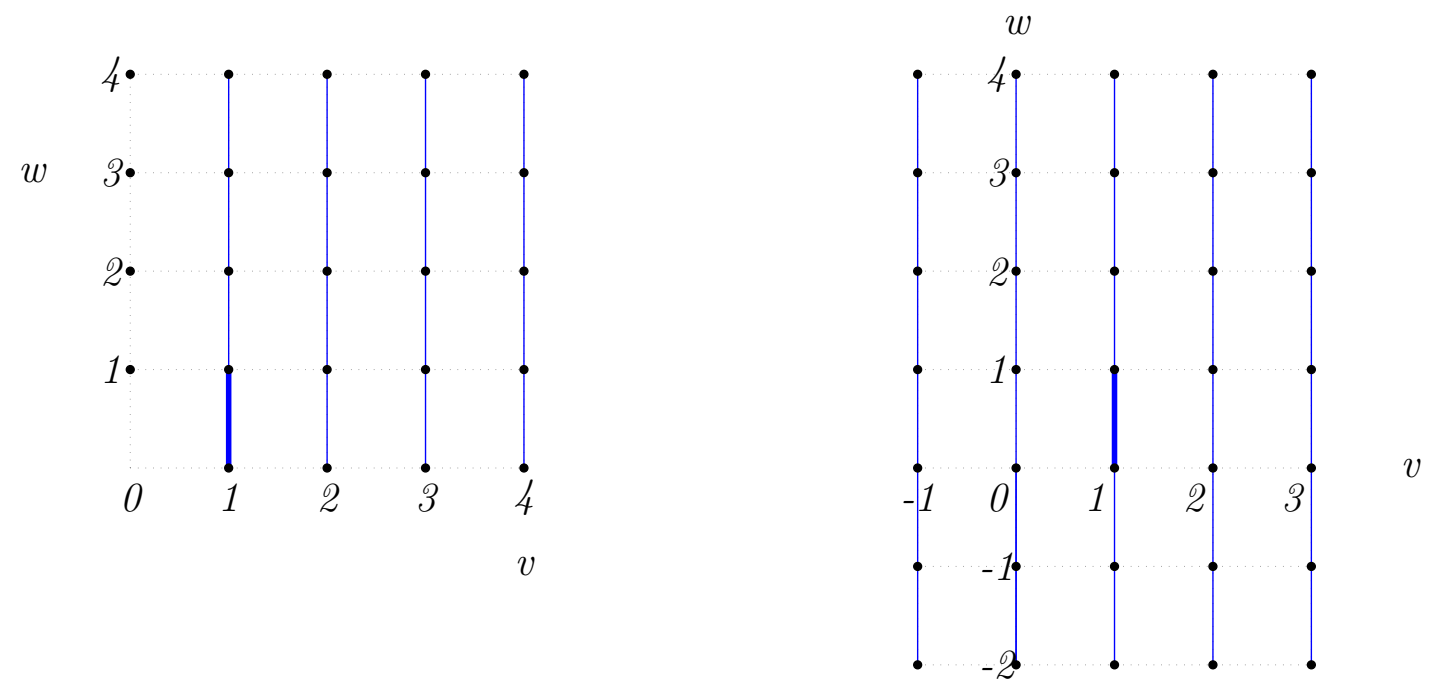

\subsection{Grothendieck Groups.}

2.2.1. Closure operator on a semigroup. An element $s$ of a semigroup $S$ is said to have infinite type (or infinite order) if $s, s^{2}, s^{3}, \ldots$ are distinct. Otherwise, $s$ is said to be torsion of type $(m, n)$ if $n$ is the least positive integer such that $x^{n}=x^{m}$ for some $0<m<n$.

If a semigroup generated by a single element is called cyclic and it is determined (up to isomorphism) by the type of its generator.

There is a pre-order $\preccurlyeq$ on a commutative (additive) semigroup $S: x \preccurlyeq y$ if and only if there is a $z \in S$ with $x+z=y$. For any subset $A$ of $S$, its closure is

$$
\bar{A}:=\{x \in S: x \preccurlyeq s \text { for some } s \in A\} .
$$

This defines a closure operator on (the power set of) $S$, since:

(1) $A \subseteq \bar{A}$

(2) $\overline{\bar{A}}=\bar{A}$,

(3) $\overline{A \cup B}=\bar{A} \cup \bar{B}$.

Note that if $A$ is a subsemigroup of $S$, then $\bar{A}$ is also a subsemigroup. A set $F$ is closed, if and only if $a \preccurlyeq b \in F$ implies $a \in F$ for all $a, b \in S$.

For any ring $R$, we denote by $\mathcal{V}(R)$ the additive commutative monoid of isomorphism classes of finitely generated projective (right) $R$-modules under direct sum. We denote the semigroup $\mathcal{V}(R) \backslash\{[0]\}$ by $\mathcal{V}^{*}(R)$. Let $\mathcal{U}(R)$ be the cyclic submonoid of $\mathcal{V}(R)$, generated 
by $[R]$, hence $\mathcal{U}^{*}(R)$ is the semigroup of isomorphism classes of finitely generated nonzero free $R$-modules. Note that $\mathcal{V}(R)=\overline{\mathcal{U}(R)}$. More generally, a finitely generated projective $R$-module $P$ is a progenerator if and only if $\overline{\langle[P]\rangle}=\mathcal{V}(R)$.

2.2.2. The Grothendieck group. The following discussion is basic and well-known [10, 7], we included to fix notation and for completeness (until the referee tells us to cut it.)

Theorem 3. [10, Theorem 1.1.3] Let $S$ be a multiplicative semigroup. There is an abelian (additive) group $G(S)$ (called the Grothendieck group of $S$ ) together with a semigroup homomorphism $\iota_{S}: S \rightarrow G(S)$, such that for any (multiplicative) abelian group $H$, and any semigroup homomorphism $f: S \rightarrow H$, there exists a unique group homomorphism $\hat{f}: G(S) \rightarrow H$ with $f=\hat{f} \circ \iota_{S}$.

Proof. The Grothendieck group of $S, G(S)$ is defined as $\mathbb{Z} S / K_{S}$ where $\mathbb{Z} S$ is a free abelian group with basis $S, K_{S}$ is a subgroup generated by $\{a+b-a b: a, b \in S\}$. The semigroup homomorphism $\iota_{S}: S \rightarrow G(S)$ maps $a \mapsto[a]=1 a+K_{S}$. Note that $\iota_{S}(a b)=[a b]=[a+b]=$ $[a]+[b]=\iota_{S}(a)+\iota_{S}(b)$

Given any abelian group $H$ and any semigroup homomorphism $f: S \longrightarrow H$, the map $\hat{f}: G(S) \longrightarrow H$ is defined as $\left[\sum n_{a} a\right] \mapsto \prod_{a \in S} f(a)^{n_{a}} . \hat{f}$ is a homomorphism since

$$
\hat{f}(a+b-a b)=f(a) f(b) f(a b)^{-1}=1 .
$$

Since $\hat{f}([a])=f \iota_{S}(a)=f(a)$, the homomorphism $\hat{f}: G(S) \rightarrow H$ is determined on the generators of $G(S)$. Hence $\hat{f}$ is unique.

We list a few relevant properties of the Grothendieck group of a semigroup.

Fact 4. Assume $S, T$ are multiplicative semigroups.

(i) $G$ is functorial : If $f: S \rightarrow T$ is a semigroup homomorphism, then there is a group homomorphism $G(f)$ induced by $f$ between the Grothendieck groups $G(S)$ and $G(T)$.

(ii) $G$ is left adjoint to the forgetful functor: For any abelian group $H$, we have a 1-1 correspondence $\operatorname{Hom}(G(S), H) \leftrightarrow \operatorname{Hom}(S, F(H))$ given by $f \mapsto \hat{f}$ and $g \mapsto g \circ \iota_{S}$ where $F(H)$ is $H$ regarded as a semigroup.

(iii) If $f: S \rightarrow T$ is onto, then $G(f): G(S) \rightarrow G(T)$ is also onto.

(iv) If $S$ is a semigroup generated by $n$ elements; then $G(S)$ is finitely generated as a group with at most $n$ generators. In particular, if $S$ is a cyclic semigroup, then $G(S)$ is a cyclic group.

(v) Assume $S$ is a cyclic semigroup, then $S$ is a finite semigroup if and only if $G(S)$ is a finite group. 
(vi) If an element $x \in S$ is torsion, then $\iota_{S}(x)$ is also torsion in $G(S)$, but the converse is not true.

Now for a given semigroup $S$, construct $S^{+}=S \cup\{*\}$ as the monoid where multiplication on $S$ extends to $S^{+}$with $* \cdot s=s=s \cdot *$ for all $s \in S^{+}$.

Lemma 5. Assume $S$ is a commutative multiplicative semigroup and $S^{+}$is the monoid defined above.

(i) If $S$ is an abelian group, $\iota_{S}: S \rightarrow G(S)$ is an isomorphism.

(ii) For any semigroup $S$, we have $G(S) \cong G\left(S^{+}\right)$.

(iii) Suppose $M$ is a commutative multiplicative monoid for which $S=M-\{1\}$ is a group. Then $G(M) \cong S$.

Proof. (i) Look at $i d: S \rightarrow S$. Now by the definition of $G(S)$, the following diagram commutes
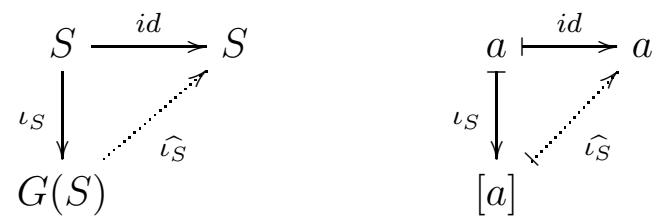

As $\widehat{\iota_{S}} \circ \iota_{S}=i d, \iota_{S}$ is $1-1$. Since $G(S)$ is generated by $\iota_{S}(S)$ and $S$ is a group, $\iota_{S}$ is onto.

(ii) $S$ is a semigroup, $S^{+}=S \cup\{*\}$ is a monoid where $* \cdot s=s=s \cdot *$ and $* \cdot *=*$ Define $\alpha: S \rightarrow S^{+}$as $s \mapsto s$, and define a monoid homomorphism $\beta: S^{+} \rightarrow G(S)$ as $\beta: s \mapsto[s]$ and $\beta: * \mapsto 0$. Then the diagram below commutes

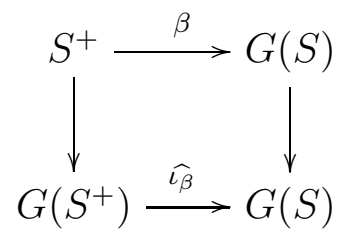

Consider the following diagrams where there exists a unique $\tilde{\iota}_{S}$ by the universality of $S^{+}$and there exists a unique $\widehat{\widetilde{\iota_{S}}}$ by the universality of $G$,
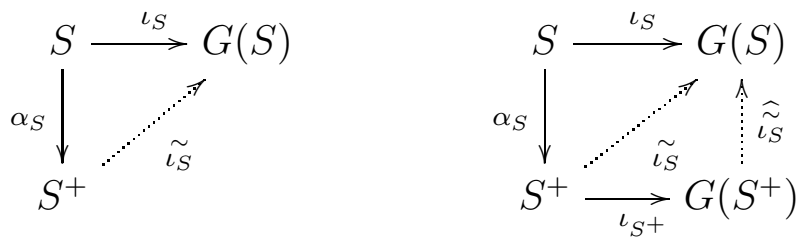
Now, in the following diagram,

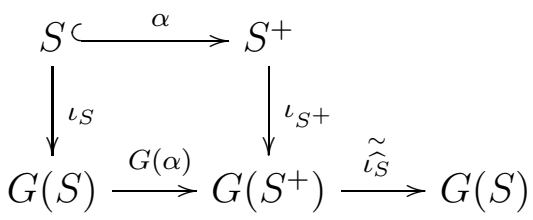

we have $[s]^{+} \mapsto[s]$ and $[s] \mapsto[s]^{+}$. Both $G(S)$ and $G\left(S^{+}\right)$are generated by $S$, $G(S)=\left\{s+K_{S}=[s]\right\}$ and $G\left(S^{+}\right)=\left\{s+K_{S^{+}}=[s]^{+}\right\}$. Further, $*=*+*-* * \in K_{S^{+}}$, so $*+K_{S^{+}}=[*]^{+}=0$. Hence, $G\left(\alpha_{S}\right) \circ \widehat{\iota_{S}}=i d_{G\left(S^{+}\right)}$and $\widehat{\iota_{S}} \circ G\left(\alpha_{S}\right)=i d_{G(S)}$. Then $G(S) \cong G\left(S^{+}\right)$.

(iii) Let $S=M-\{1\}$, then $M=S^{+}$. The result follows from the parts above.

Some (counter)examples:

Example 6. (i) $\iota: S \rightarrow G(S)$ is not necessarily one-to-one. For instance:

Let $S=<a: n a=m a>$ where $n, m$ are distinct positive integers with $n>m>1$. Then $G(S)=\mathbb{Z} /(n-m) \mathbb{Z}$ and $|S|=n-1>|G(S)|=n-m$. Hence, $\iota_{S}$ is not 1-1.

(ii) If $f: S \rightarrow T$ is 1-1, then $G(f): G(S) \rightarrow G(T)$ may not be 1-1: Consider the additive semigroups $S=\mathbb{N}$ and $T=<v, w \mid v=v+w>$. ( $T$ is the semigroup of Example [.) Let $f: S \rightarrow T$ be the semigroup monomorphism defined as $f(1)=w$, $f(n)=$ nw. Then both $G(S)$ and $G(T)$ are $\mathbb{Z}$, but the group homomorphism $G(f)$ induced by $f$ between the Grothendieck groups $G(S)$ and $G(T)$ is the zero map which is not injective. However, if we take $S=\mathcal{U}(R)$ and $T=\mathcal{V}(R)$ for any ring $R$, then $G(f): G(\mathcal{U}(R)) \rightarrow \mathcal{K}_{0}(R)$ is also a monomorphism which we will prove shortly in Proposition 7 .

(iii) Let $a \in T$ be an element with infinite order, then it is possible that $\iota($ a) has finite order: In the example above, $w \in T$ is not torsion and $\iota(v)=\iota(v+w)=\iota(v)+\iota(w)$ in $G(T)$. Hence, $\iota(w)=0$ and $\iota(w)$ is torsion in $G(T)=\mathbb{Z}$.

We will proceed with an alternative construction of the Grothendieck group of a commutative semigroup which will also be used in the sequel. Let $S$ be a non-empty commutative semigroup, then the relation $\sim$ on $S \times S$, defined as $(a, b) \sim(c, d)$ if and only if there exists $x \in S$ such that $a d x=b c x$, is an equivalence relation. The set of all equivalence classes of $S \times S$ is denoted by $E(S)$, and the operation - on $E(S)$ is defined as $[(a, b)] \cdot[(c, d)]=[(a c, b d)]$. For any $\delta \in S,[(\delta, \delta)]$ is the identity element of $\cdot$, and $[(a, b)]$ is the inverse element for $[(b, a)]$. Hence, $E(S)$ is an abelian group where commutativity of $E(S)$ follows from the commutativity of $S$. Fix an $x \in S$, define $\phi_{x}: S \rightarrow E(S)$ which maps $a \in S$ to $[(a x, x)] \in E(S)$. Now, 
$\phi_{x}$ is a semigroup homomorphism. For any $x, y \in S, \phi_{x}=\phi_{y}$; hence $\phi$ is defined independent of the choice of $x \in S$. Also, consider the map $\hat{\phi}: G(S) \rightarrow E(S)$ induced by $\phi$ which maps $a+K_{S} \mapsto[(a x, x)]$.

Now, define $f: E(S) \rightarrow G(S)$ as $[(a, b)] \mapsto a-b+K_{S}$. So $f$ is clearly a group homomorphism. It is straightforward to show that $f \circ \hat{\phi}$ and $\hat{\phi} \circ f$ are identity maps, i.e.,

$[a, b] \mapsto a-b+K_{S} \mapsto[a x, x][b x, x]^{-1}=[a x, x][x, b x]=\left[a x^{2}, b x^{2}\right]=[a, b]$ and also,

$a+K_{S} \mapsto[a x, x] \mapsto a x-x+K_{S}=a+x-x+K_{S}=a+K_{S}$. So $E(S), G(S)$ are (naturally and canonically) isomorphic groups. We are going to identify $E(S)$ with $G(S)$ and use them interchangibly from now on.

Note that $\left(\mathcal{V}^{*}(R)\right)^{+}=\mathcal{V}(R)$ and recall that $\mathcal{K}_{0}(R)$ is defined as the Grothendieck group of the monoid $\mathcal{V}(R)$.

Compare the following proposition with Example 6 part (iii).

Proposition 7. For any ring $R$, consider $\mathcal{U}(R)$ and $\mathcal{V}(R)$ as defined above, and $f$ the inclusion map, then the induced map $G(f): G(\mathcal{U}(R)) \rightarrow \mathcal{K}_{0}(R)$ is injective.

Proof. Let us take an element $m[R]_{G(\mathcal{U}(R))}$ for some $n \in \mathbb{Z}$, from the kernel of $G(f)$. WLOG we may choose $n \in N$ as $G(\mathcal{U}(R))$ is a group. Then $0=G(f)\left(m[R]_{G(\mathcal{U}(R))}\right)=m[R]_{G(T)}$. We have the following commutative diagram
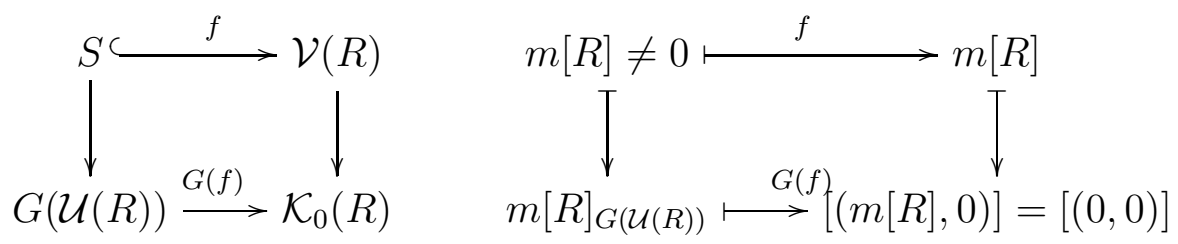

We want to show that $m[R]_{G(\mathcal{U}(R))}=0$.

$m[R]+0+[X]=0+[X]$ in $\mathcal{V}(R)$, that is, $R^{m} \oplus X \cong X$ for some finitely generated projective module over $R$. Then there exists an $R$-module $Y$ such that $X \oplus Y=R^{n}$ for some $n \in \mathbb{N}$. $R^{m} \oplus X \oplus Y \cong X \oplus Y$, i.e., $R^{m} \oplus R^{n} \cong R^{n}$, so $(n+m)[R]=n[R]$ in $\mathcal{V}(R)$, hence in $\mathcal{U}(R)$. Chasing the diagram we get $(n+m)[R]_{G(\mathcal{U}(R))}=n[R]_{G(\mathcal{U}(R))}$ in $G(\mathcal{U}(R))$. Since $G(\mathcal{U}(R))$ is a group we have inverses and get $m[R]_{G(\mathcal{U}(R))}=0$. Hence, $G(f)$ is a monomorphism.

For the isomorphism class of the free module of rank one $[R] \in \mathcal{V}(R)$, we use the following notation. Let $\iota([R]):=[R]_{\mathbb{Z}} \in \mathcal{K}_{0}(R)$ and $[R]_{\mathbb{Q}}:=[R]_{\mathbb{Z}} \otimes_{\mathbb{Z}} 1 \in \mathcal{K}_{0}(R) \otimes_{\mathbb{Z}} \mathbb{Q}$. If an element $[P]$ is torsion in $\mathcal{V}^{*}(R)$, then $\iota([P])$ has finite order in $\mathcal{K}_{0}(R)$. 
2.2.3. Graph Semigroups. $\Gamma=(E, V, s, t)$ will denote a digraph with vertex set $V$, edge set $E$, source and target functions $s, t: E \rightarrow V$. A sink is a vertex $v$ for which the outgoing edge set $s^{-1}(v)$ is empty and a source is a vertex $v$ for which the incoming edge set $t^{-1}(v)$ is empty. In particular, an isolated vertex is both a source and a sink. Throughout this note we will assume $\Gamma$ is a finite digraph, that is both $V$ and $E$ are finite.

A separated graph $(\Gamma, \Pi)$ is a digraph $\Gamma$ together with a partition $\Pi$ of $E$ which is finer than $\left\{s^{-1}(v) \mid v \in V, v\right.$ not a sink $\}$.

For a given triple $(\Gamma, \Pi, \Lambda)$ where $\Lambda \subseteq \Pi, S(\Gamma, \Pi, \Lambda)$ is defined to be the commutative semigroup generated by $\Omega:=V \sqcup(\Pi \backslash \Lambda)$ subject to the relations $\left\{R_{X}\right\}_{X \in \Pi}$ such that

$$
\begin{gathered}
s X=\sum_{e \in X} t(e) \quad \text { for every } X \in \Lambda, \text { and } \\
s X=X+\sum_{f \in X} t(f) \quad \text { for every } X \in \Pi \backslash \Lambda .
\end{gathered}
$$

The Grothendieck group of $\mathbb{N} \Omega \backslash\{\mathbf{0}\}$ is $\mathbb{Z} \Omega$. The Grothendieck group of $S(\Gamma, \Pi, \Lambda)$ is $\mathbb{Z} \Omega / f\left(R_{X}\right)$ where $X \in \Pi$ which we will denote as $S_{\mathbb{Z}}(\Gamma, \Pi, \Lambda)$. Notice also that $f\left(R_{X}\right)$ is exactly the $\mathbb{Z}$-span of the relations $R_{X}$.

Example 8. Consider the following separated graph $\Gamma$ with vertex set $V=\{v, w\}$ and edge set $E=\left\{e_{1}, e_{2}, f_{1}, f_{2}, f_{3}, f_{4}, f_{5}\right\}$ with $s(e)=v$ and $t(e)=w$ for all $e \in E, \Pi=\{X, Y\}$ where $X=\left\{e_{1}, e_{2}\right\}, Y=\left\{f_{1}, f_{2}, f_{3}, f_{4}, f_{5}\right\}$ and $\Lambda=\Pi$. We marked the edges in $X$ with red, and the edges in $Y$ with blue in the graph. The set $\Omega=V$ and $v=2 w$ and $v=5 w$ are the relations on $\mathbb{N} \Omega \backslash\{\mathbf{0}\}$.

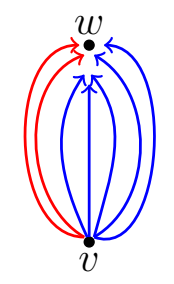

The graph semigroup $S(\Gamma, \Pi)=S(\Gamma, \Pi, \Pi)$ is the semigroup of Example 1.

Example 9. Consider the following digraph $\Gamma$ :

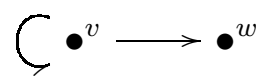

where $\Pi=\{E\}$ and $\Lambda=\Pi$. Now $\Omega=V$ and $v=v+w$ is the only relation. The graph semigroup is the semigroup of Example 2 . 


\section{Separated Cohn-Leavitt Path Algebras and IBN}

We can rephrase IBN property for a ring $R$ in terms of $\mathcal{V}^{*}(R)$ as follows: $R$ has IBN if and only if for every pair of distinct positive integers $m \neq m^{\prime}$ we have $m[R] \neq m^{\prime}[R]$ as elements of $\mathcal{V}^{*}(R)$. Hence, $[R]$ has infinite order in $\mathcal{V}^{*}(R)$ if and only if $R$ is IBN. Moreover, $[R]$ has infinite order in $\mathcal{U}(R)$. For any ring $R$, the property of having IBN is detected by $\mathcal{K}_{0}(R)$.

Proposition 10. 4, Corollary 5.7] For any ring $R$, the followings are equivalent:

(1) $R$ has IBN;

(2) $\mathcal{U}(R)$ is infinite;

(3) $[R]_{\mathbb{Q}}$ is nonzero in $\mathcal{K}_{0}(R) \otimes \mathbb{Q}$.

Proof. Now, $R$ has IBN if and only if $\mathcal{U}(R)$ is infinite is clear from the definitions. By Proposition 7, $G(\mathcal{U}(R)) \rightarrow \mathcal{K}_{0}(R)$ induced from the semigroup inclusion $\mathcal{U}(R) \rightarrow \mathcal{V}(R)$ is injective. Now, if $\mathcal{U}(R)$ is an infinite cyclic semigroup then its Grothendieck group $G(\mathcal{U}(R))$ is an infinite cyclic group generated by $[R]$ by Fact 4 parts (iv) and (四). So $[R]_{\mathbb{Z}}$ in $\mathcal{K}_{0}(R)$ also has infinite order. Hence $[R]_{\mathbb{Q}}$ in $\mathcal{K}_{0}(R) \otimes \mathbb{Q}$ is nonzero.

If $\mathcal{U}(R)$ is a finite cyclic semigroup of type $(m, n)$, then $[R]_{\mathbb{Z}}$ in $G(\mathcal{U}(R))$ generates a finite cyclic group of order $n-m$, so $[R]_{\mathbb{Z}}$ in $\mathcal{K}_{0}(R)$ also has order $n-m(>0)$ and its image in $\mathcal{K}_{0}(R) \otimes \mathbb{Q}$ is 0.

Definition 11. For a given triple $(\Gamma, \Pi, \Lambda)$, the separated Cohn-Leavitt path algebra over the field $K$, denoted by $C L_{K}(\Gamma, \Pi, \Lambda)$, is the algebra which is generated by the sets $\{v \mid v \in V\},\left\{e, e^{*} \mid e \in E\right\}$, which satisfy the following relations:

(V) $v w=\delta_{v, w} v$ for all $v, w \in V$,

(E1) $s(e) e=e t(e)=e$ for all $e \in E$,

(E2) $t(e) e^{*}=e^{*} s(e)=e^{*}$ for all $e \in E$, and

(CK1) For all $X \in \Pi, e^{*} f=\delta_{e f} t(e)$ for every $e, f \in X$.

(CK2) For all $X \in \Lambda, s X=\sum_{\{e \in X\}} e e^{*}$ for every $e \in X$.

For ease of notation we will drop $K$, and use $C L(\Gamma, \Pi, \Lambda)$.

If $\Lambda=\Pi$, then $L(\Gamma, \Pi):=C L(\Gamma, \Pi, \Pi)$ is the separated Leavitt path algebra over the separated graph $(\Gamma, \Pi)$;

if $\Lambda=\emptyset$, then $C(\Gamma, \Pi):=C L(\Gamma, \Pi, \emptyset)$ is the separated Cohn path algebra over the separated graph $(\Gamma, \Pi)$;

if $\Lambda=\Pi=\left\{s^{-1}(v) \mid v \in V, v\right.$ not a sink $\}$, then $L(\Gamma):=C L(\Gamma, \Pi, \Pi)$ is the Leavitt path algebra over the graph $\Gamma$, and 
if $\Lambda=\emptyset$ and $\Pi=\left\{s^{-1}(v) \mid v \in V, v\right.$ not a sink $\}$, then $C(\Gamma):=C L(\Gamma, \Pi, \emptyset)$ is the Cohn path algebra over the graph $\Gamma$.

The connection between the graph semigroup and the non-stable $\mathcal{K}$-theory of the separated Cohn-Leavitt path algebra $C L(\Gamma, \Pi, \Lambda)$ is provided by the following lemma.

Lemma 12. For a given triple $(\Gamma, \Pi, \Lambda)$, let $L$ denote $C L(\Gamma, \Pi, \Lambda)$. Then

$$
\begin{gathered}
(s X) L \cong \bigoplus_{e \in X} t(e) L \quad \text { for } X \in \Lambda \\
(s Y) L \cong \bigoplus_{e \in Y} t(e) L \bigoplus\left(s Y-\sum_{e \in Y} e e^{*}\right) L \quad \text { for } Y \in \Pi \backslash \Lambda .
\end{gathered}
$$

Proof. Notice that $s Y-\sum_{e \in Y} e e^{*}$ is an idempotent, as

$$
\begin{gathered}
\left(s Y-\sum_{e \in Y} e e^{*}\right)\left(s Y-\sum_{e \in Y} e e^{*}\right)=s Y-s Y \sum_{e \in Y} e e^{*}-\left(\sum_{e \in Y} e e^{*}\right) s Y+\left(\sum_{e \in Y} e e^{*}\right)^{2} \\
s Y-\sum_{e \in Y} e e^{*}-\left(\sum_{e \in Y} e e^{*}\right)+\left(\sum_{e \in Y} e e^{*}\right)=s Y-\sum_{e \in Y} e e^{*}
\end{gathered}
$$

by using the (CK2) relation. Hence, $\left(s Y-\sum_{e \in Y} e e^{*}\right) L$ is a cyclic projective $L$-module, so is $v L$ for every vertex $v$ of $\Gamma$.

For $X \in \Lambda$, define the following maps $f_{1}:(s X) L \rightarrow \bigoplus_{e \in X} t(e) L$ as $m \mapsto\left(e^{*} m\right)_{e \in X}$ and $f_{2}: \bigoplus_{e \in X} t(e) L \rightarrow(s X) L$ as $\left(m_{e}\right)_{e \in X} \mapsto \sum_{e \in X} e m_{e}$ where $f_{1} \circ f_{2}$ and $f_{2} \circ f_{1}$ are both the identity functions. Similarly, define $g_{1}:(s Y) L \rightarrow\left(\bigoplus_{e \in Y} t(e) L\right) \bigoplus\left(s Y-\sum_{e \in Y} e e^{*}\right) L$ as $m \mapsto\left(\left(e^{*} m\right)_{e \in Y},\left(s Y-\sum_{e \in Y} e e^{*}\right) m\right)$ and $g_{2}:\left(\bigoplus_{e \in X} t(e) L\right) \bigoplus\left(s Y-\sum_{e \in Y} e e^{*}\right) L \rightarrow(s Y) L$ as $\left(\left(m_{e}\right)_{e \in Y}, m_{Y}\right) \mapsto \sum_{e \in Y} e m_{e}+m_{Y}$ where $g_{1} \circ g_{2}$ and $g_{2} \circ g_{1}$ are also the identity functions.

For a given triple $(\Gamma, \Pi, \Lambda)$, let $L$ denote $C L(\Gamma, \Pi, \Lambda)$. Then there exists a semigroup homomorphism $\alpha: S(\Gamma, \Pi, \Lambda) \rightarrow \mathcal{V}(L)$ that maps $v \mapsto[v L]$ and $Y \mapsto\left[\left(s Y-\sum_{e \in Y} e e^{*}\right) L\right]$ for $v \in V$, and $Y \in \Pi \backslash \Lambda$. The map $\alpha$ defined on the generators of $\mathbb{N} \Omega$ can be extended additively. We can show that $\alpha$ preserves the relations on $S(\Gamma, \Pi, \Lambda)$ by Lemma 12. Hence, $\alpha$ is a semigroup homomorphism. In fact, this homomorphism $\alpha$ is an isomorphism as proved in [3, Theorem 4.3] which we will use, but not prove here.

Now, we are ready to prove our main result.

Theorem 13. For a given triple $(\Gamma, \Pi, \Lambda)$, let $L$ denote the Cohn-Leavitt path algebra, $C L(\Gamma, \Pi, \Lambda)$ over the triple. Then $L$ is IBN if and only if $\sum_{v \in V} v$ is not in the $\mathbb{Q}$-span of the relations $\left\{s X-\sum_{e \in X} t e\right\}_{X \in \Lambda}$ in $\mathbb{Q} \Omega$. 
Proof. In the following diagram of commutative additive semigroups,

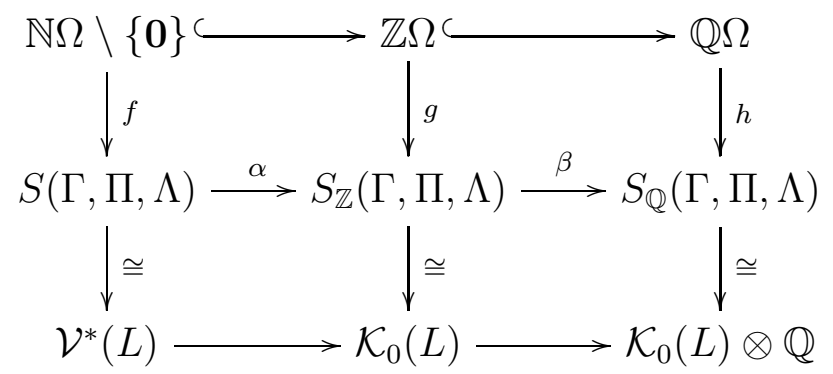

$f, g, h$ are epimorphisms with $\operatorname{Ker}(g)=\mathbb{Z}-\operatorname{span}\left\{R_{X}\right\}_{X \in \Pi}$ and $\operatorname{Ker}(h)=\mathbb{Q}-\operatorname{span}\left\{R_{X}\right\}_{X \in \Pi}$ . Now, $S(\Gamma, \Pi, \Lambda) \cong \mathcal{V}^{*}(L)$ by [3, Theorem 4.3] and since taking Grothendieck group and tensoring with $\mathbb{Q}$ are both functorial $S_{\mathbb{Z}}(\Gamma, \Pi, \Lambda) \cong \mathcal{K}_{0}(L)$ and $S_{\mathbb{Q}}(\Gamma, \Pi, \Lambda) \cong \mathcal{K}_{0}(L) \otimes \mathbb{Q}$.

$L$ has IBN if and only if $\mathcal{U}(L)$ is infinite if and only if $[L]$ has infinite order in $\mathcal{U}(L)$ by Proposition 10. Moreover, $\mathcal{U}(L)$ is cyclic, so $\mathcal{U}(L) \cong \mathbb{N}$ and $G(\mathcal{U}(L)) \cong \mathbb{Z}$. By Proposition 7 , as $G(\mathcal{U}(L))$ is embedded in $\mathcal{K}_{0}(L) ;[L]_{\mathbb{Z}}$ also has infinite order in $\mathcal{K}_{0}(L)$. This is identical to: $[L]_{\mathbb{Q}}$ is non-zero in $\mathcal{K}_{0}(L) \otimes \mathbb{Q}$. On the other hand, by [3, Theorem 4.3], $S(\Gamma, \Pi, \Lambda) \cong \mathcal{V}(L)$. $\left[\sum_{v \in V} v\right]$ is non-zero in $G(S(\Gamma, \Pi, \Lambda)) \otimes \mathbb{Q}$. Equivalently, $\sum_{v \in V} v$ is not in the $\mathbb{Q}$-span of the relations $\left\{R_{X}\right\}_{X \in \Pi \text {. }}$

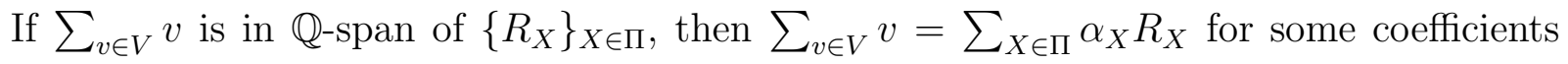
$\alpha_{X} \in \mathbb{Q}$. However, we are going to show that the coefficient of $R_{Y}$, for any $Y \in \Pi \backslash \Lambda$ has to be zero.

For any fixed $Y \in \Pi \backslash \Lambda$, consider the projection map $p r_{Y}: \mathbb{Q} \Omega \rightarrow \mathbb{Q}$. Note that $\mathbb{Q} \Omega=$ $\mathbb{Q} V \oplus \mathbb{Q}(\Pi \backslash \Lambda)$, so $\operatorname{pr}_{Y}\left(\sum_{v \in V} v\right)=0$, hence $\operatorname{pr}_{Y}\left(\sum_{X \in \Pi} \alpha_{X} R_{X}\right)=0$. Since $\operatorname{pr}_{Y}\left(R_{X}\right)=\delta_{X Y}$, then in this sum $X \neq Y$. Therefore, $X \notin \Pi \backslash \Lambda$, i.e., $X \in \Lambda$.

The original proof of Theorem 13 was more geometric. It was presented by the first named author at the CIMPA Research School Izmir, Turkey, in July 2015. (The videos of these talks have been publicly available as instructed by CIMPA.) We will sketch this geometric proof below.

Second Proof. If $\sum_{v \in V} v$ is in the $\mathbb{Q}$-span of the relations of the semigroup of $(\Gamma, \Pi, \Lambda)$, then there exists $k \in \mathbb{Z}$, so that $k \sum_{v \in V} v$ is in the $\mathbb{Z}$-span of the relations $R_{X}$, for $X \in \Pi$. Hence, there is a path from $\sum_{v \in V} v$ to $(k+1)\left(\sum_{v \in V} v\right)$ in the $\mathbb{Z} \Omega$ representation of $S(\Gamma, \Pi, \Lambda)$. Now, if this path is totally contained in $\mathbb{N} \Omega \backslash\{\mathbf{0}\}$, then $L^{1} \cong L^{k+1}$ where $L$ is the separated CohnLeavitt path algebra over $(\Gamma, \Pi, \Lambda)$. If not, as the geometric representation of $S(\Gamma, \Pi, \Lambda)$ is translation invariant, we can move the path from $\mathbb{Z} \Omega$ to $\mathbb{N} \Omega \backslash\{\mathbf{0}\}$ translating by $t \sum_{v \in V} v$ by sufficiently large $t$. Hence, we obtain a path from $(1+t)\left(\sum_{v \in V} v\right)$ to $(k+t)\left(\sum_{v \in V} v\right)$ in the $\mathbb{N} \Omega \backslash\{\mathbf{0}\}$ representation of $S(\Gamma, \Pi, \Lambda)$. Again, $L^{1+t} \cong L^{k+t}$. Thus, $C L(\Gamma, \Pi, \Lambda)$ is not IBN. 
Conversely, if $L=C L(\Gamma, \Pi, \Lambda)$ is not IBN, then there exist distinct $k, l \in \mathbb{N}$ with $L^{k} \cong L^{l}$. Then there is a path from $k\left(\sum_{v \in V} v\right)$ to $l\left(\sum_{v \in V} v\right)$ in the $\mathbb{N} \Omega \backslash\{\mathbf{0}\}$ representation of $S(\Gamma, \Pi, \Lambda)$. (or both vectors are in the same path component) Then $(k-l) \sum_{v \in V} v$ is in the $\mathbb{Z}$-span of the relations of the semigroup of $(\Gamma, \Pi, \Lambda)$, hence $\sum_{v \in V} v$ is in the $\mathbb{Q}$-span of

the relations $R_{X}$ for $X \in \Pi$. In fact, $\sum_{v \in V} v$ is in the $\mathbb{Q}$-span of the relations $R_{X}$ for $X \in \Lambda$ can be shown as in the last paragraph of the first proof.

Now, an immediate corollary to the main result is that any separated Cohn path algebra has IBN. This generalizes the fact that any Cohn path algebra has IBN which was proven in [2, Theorem 9].

Corollary 14. Any separated Cohn path algebra has IBN.

\section{Corner Subalgebras of Separated Cohn-leavitt Path Algebras and IBN}

The next theorem shows that the non-stable $\mathcal{K}$-theory of a corner ring is given explicitly in terms of the non-stable $\mathcal{K}$-theory of the ring.

Theorem 15. Assume $R$ is a ring and $\alpha \in R$ is an idempotent. Then the semigroup homomorphism induced by $-\otimes_{\alpha R \alpha} \alpha R$ from $\mathcal{V}(\alpha R \alpha)$ into $\mathcal{V}(R)$ is 1-1. Moreover,

$$
\mathcal{V}(\alpha R \alpha) \cong \overline{<[\alpha R]>} \subseteq \mathcal{V}(R) .
$$

Proof. Assume $R$ is a ring (not necessarily unital) and $\alpha \in R$ is an idempotent. $v R$ is a projective right $R$-module. Let $\alpha R \alpha$ be the associated corner subring of $R$. Note that $\alpha$ is the unit element in $\alpha R \alpha$, so $\alpha R \alpha$ is a unital ring. However, $\alpha R \alpha$ is not a unital subring of $R$, as $\alpha$ is not the unit of $R$ unless $\alpha R \alpha=R$. $\alpha R$ is finitely generated $(\alpha R \alpha, R)$-bimodule and as a right $R$-module it is finitely generated (in fact, cyclic) projective.

Our aim is to prove that $\mathcal{V}(\alpha R \alpha)$ is embedded into $\mathcal{V}(R)$. Consider $\mathcal{M}_{\alpha R \alpha}$ as the category of right $\alpha R \alpha$-modules, $\mathcal{M}_{R}$ as the category of right $R$-modules and $F$ as the functor $-\otimes_{\alpha R \alpha}$ $\alpha R$ from $\mathcal{M}_{\alpha R \alpha}$ to $\mathcal{M}_{R}$. Now, the functor $F$ induces a semigroup homomorphism $f$ from $\mathcal{V}(\alpha R \alpha)$ to $\mathcal{V}(R)$ as for any isomorphism class of finitely generated projective $R$-module $P$,

$$
[P] \stackrel{f}{\mapsto}\left[P \otimes_{\alpha R \alpha} \alpha R\right] .
$$

Also, define $G$ as the functor $\operatorname{Hom}_{R}(\alpha R,-)$ from $\mathcal{M}_{R}$ to $\mathcal{M}_{\alpha R \alpha}$. Notice that $\alpha R$ is mapped to $\operatorname{Hom}_{R}(\alpha R, \alpha R)$ which is isomophic to $\alpha R \alpha$ as a $\alpha R \alpha$-module. We can also show that the functor $H: \mathcal{M}_{R} \rightarrow \mathcal{M}_{\alpha R \alpha}$ defined as $M \mapsto M \alpha$ for any $R$-module $M$, is equivalent to the functor $G$. Hence, replacing $G$ with $H$, for any projective $R$-module $P$ we get

$$
P \stackrel{F}{\mapsto} P \otimes_{\alpha R \alpha} \alpha R \stackrel{H}{\mapsto}(P \otimes \alpha R) \alpha .
$$


Take $\alpha R \alpha \hookrightarrow \alpha R$ inclusion map, and tensor with a projective module $P$, that is:

$$
P \otimes \alpha R \alpha \stackrel{\beta}{\rightarrow} P \otimes \alpha R
$$

As $P$ is projective, it is flat; so $\beta$ is 1 - 1 . Moreover, $P \cong P \otimes \alpha R \alpha \cong \beta(P \otimes \alpha R \alpha)=(P \otimes \alpha R) \alpha$. We proved that $(P \otimes \alpha R) \alpha \cong P$, and conclude that their isomophism classes in $\mathcal{V}(R)$ are the same. The semigroup homomorphism $h$, induced by the functor $H$ gives

$$
[P] \stackrel{f}{\mapsto}\left[P \otimes_{\alpha R \alpha} \alpha R\right] \stackrel{h}{\mapsto}[P] .
$$

Thus, the semigroup homomorphism $f$ is $1-1$.

In particular,

$$
<[\alpha R \alpha]>=\mathcal{U}(\alpha R \alpha) \cong<[\alpha R]>\subseteq \mathcal{V}(R) .
$$

For the last statement, we will show that the closure of $\langle[\alpha R]>$ in $\mathcal{V}(R)$ is isomorphic to the closure of $\mathcal{U}(\alpha R \alpha)$ in $\mathcal{V}(\alpha R \alpha)$.

Consider a (right) $R$-module $P$ where $P \oplus Q \cong(\alpha R)^{k}$ for some positive integer $k$ and $R$ module $Q$, i.e., $[P]$ is an element of $\overline{\langle[\alpha R]>}$ in $\mathcal{V}(R)$. We can identify $P$ with its isomorphic copy as a submodule in $(\alpha R)^{k}$.

Consider the maps $\mu: \operatorname{Hom}_{R}(\alpha R, P) \otimes \alpha R \rightarrow P$, and $\eta: P \rightarrow \operatorname{Hom}_{R}(\alpha R, P) \otimes \alpha R$ mapping $\mu: f \otimes x \mapsto f x$ and $\eta: y \mapsto \sum_{j=1}^{k} \phi_{j} \otimes \pi_{j} y$ where $\pi_{j}: P \rightarrow \alpha R$ is the (restriction of the) projection map to the $j^{\text {th }}$ component and $\phi_{j}: \alpha R \rightarrow P$ defined as the composition of the inclusion of $\alpha R$ into $(\alpha R)^{k}$ as the $j^{\text {th }}$ coordinate with the projection from $(\alpha R)^{k}$ to $P$.

Note that $\pi_{j} \circ f \in \operatorname{End}_{R}(\alpha R) \cong \alpha R \alpha$ and $\operatorname{Hom}_{R}(\alpha R, P)$ is a right $\operatorname{End}_{R}(\alpha R)$-module. (All tensor products are over $\alpha R \alpha$.) Further, $\left(\sum_{j=1}^{k} \phi_{j} \pi_{j}\right)$ is the identity map on $P$. We get

$$
\begin{gathered}
\eta \mu(f \otimes x)=\sum_{j=1}^{k} \phi_{j} \otimes \pi_{j} f x=\left(\sum_{j=1}^{k} \phi_{j} \pi_{j}\right) f \otimes x=f \otimes x \text { and } \\
\mu \eta(y)=\sum_{j=1}^{k} \phi_{j} \otimes \pi_{j} y=\sum_{j=1}^{k} \phi_{j} \pi_{j} y=y .
\end{gathered}
$$

We need to show that $\operatorname{Hom}_{R}(\alpha R, P)$ is finitely generated projective. As $P \oplus Q \cong(\alpha R)^{k}$ for some positive integer $k$ and $R$-module $Q$, applying the $\operatorname{Hom}_{R}(\alpha R,-)$ functor we get

$$
\operatorname{Hom}_{R}(\alpha R, P) \oplus \operatorname{Hom}_{R}(\alpha R, Q) \cong \operatorname{Hom}_{R}\left(\alpha R,(\alpha R)^{k}\right)=\left(\operatorname{Hom}_{R}(\alpha R, \alpha R)\right)^{k} \cong(\alpha R \alpha)^{k} .
$$

Hence, $\operatorname{Hom}_{R}(\alpha R,-)$ induces an isomorphism from $\overline{<[\alpha R]>}$ to $\mathcal{V}(\alpha R \alpha)$.

Corollary 16. Assume $R$ is a ring and $\alpha \in R$ is an idempotent. Then for $m<n$, $(\alpha R \alpha)^{m} \cong(\alpha R \alpha)^{n}$ as $\alpha R \alpha$-modules if and only if $(\alpha R)^{m} \cong(\alpha R)^{n}$ as $R$-modules. Hence, 
$\alpha R \alpha$ has IBN if and only if $[\alpha R]$ has infinite order in $\mathcal{V}(R)$. Otherwise, $\alpha R \alpha$ is non-IBN of type $(m, n)$ if and only if $[\alpha R]$ is torsion of type $(m, n)$ in $\mathcal{V}(R)$.

Remark 17. Note that Corollary 16 can be proven directly as:

$$
H \mathrm{~m}_{R}\left((\alpha R)^{m},(\alpha R)^{n}\right) \cong M_{n \times m}(\alpha R \alpha) \cong \operatorname{Hom}_{\alpha R \alpha}\left((\alpha R \alpha)^{m},(\alpha R \alpha)^{n}\right) .
$$

However, this direct proof does not give $\mathcal{V}(\alpha R \alpha)$ as a submonoid of $\mathcal{V}(R)$.

Theorem 18. Assume $L$ is the Cohn-Leavitt path algebra of $(\Gamma, \Pi, \Lambda)$ and $\alpha \in L$ is an idempotent of the form $\alpha=\sum_{v \in H} v$ where $H$ is a subset of the vertex set $V$. If $\alpha$ is not in the span of the relations $\left\{s X-\sum_{e \in X} \text { te }\right\}_{X \in \Lambda}$ in $\mathbb{Q} \Omega$, then $\alpha R \alpha$ has IBN.

Proof. Let $R_{X}:=s X-\sum_{e \in X}$ te. If $\alpha=\sum_{v \in H} v$ is in $\mathbb{Q}$-span of $\left\{R_{X}\right\}_{X \in \Pi}$, then $\alpha=\sum_{v \in H} v$ is in $\mathbb{Q}$-span of $\left\{R_{X}\right\}_{X \in \Lambda}$ as proven in the proof of Theorem 13, So we can assume $\alpha=$ $\sum_{v \in H} v$ is not in the $\mathbb{Q}$-span of the relations $\left\{R_{X}\right\}_{X \in \Pi}$. Equivalently, $\left[\sum_{v \in H} v\right]_{\mathbb{Q}}$ is non-zero in $G(S(\Gamma, \Pi, \Lambda)) \otimes \mathbb{Q}$. On the other hand, by [3, Theorem 4.3], $S(\Gamma, \Pi, \Lambda) \cong \mathcal{V}(L)$, and $\left[\oplus_{v \in H} v L\right]_{\mathbb{Q}}$ is non-zero in $\mathcal{K}_{0}(L) \otimes \mathbb{Q}$. Equivalently, $\left[\oplus_{v \in H} v L\right]_{\mathbb{Z}}$ also has infinite order in $\mathcal{K}_{0}(L)$ if and only if $\left[\oplus_{v \in H} v L\right]$ has infinite order in $\mathcal{V}(L)$. Then, $[\alpha L \alpha]$ has infinite order in $\mathcal{V}(\alpha L \alpha)$ if and only if $[\alpha L \alpha]$ has infinite order in $\mathcal{U}(\alpha L \alpha)$ if and only if $\alpha L \alpha$ has IBN by Proposition 10.

Example 19. Consider the Toeplitz algebra $L$ as the Leavitt path algebra of the following graph $\Gamma$ of Example 9

Consider the following diagram,

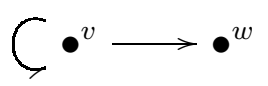

$$
\begin{gathered}
S(\Gamma) \longrightarrow S_{\mathbb{Z}}(\Gamma) \longrightarrow S_{\mathbb{Q}}(\Gamma) \\
{[v] \longmapsto[v]_{\mathbb{Z}} \longmapsto[v]_{\mathbb{Q}} \neq 0} \\
{[w] \longmapsto[w]_{\mathbb{Z}}=0 \longmapsto 0}
\end{gathered}
$$

Now, $[v] \in S(\Gamma)$ is not torsion, and neither is $[v]_{\mathbb{Z}}$ or $[v]_{\mathbb{Q}}$. Hence, $v$ is not in the $\mathbb{Q}$-span of the relation $R_{1}: v=v+w$, then by Theorem 18, vLv has IBN.

However, $[w] \in S(\Gamma)$ is not torsion, so by Corollary [16, wLw has IBN. But $[w]_{\mathbb{Z}}=0 \in$ $S_{\mathbb{Z}}(\Gamma)$ is torsion. Hence, $w$ is in the $\mathbb{Q}$-span of the relation $R_{1}: v=v+w$, and Theorem 18 is inconclusive. This example shows that the converse of Theorem 18 is not true. 
In [2], an example of two Morita equivalent rings, one having IBN and the other non-IBN is constructed. Next example provides Morita equivalent rings which are non-IBN, but are of different types.

Example 20. Consider the following separated graph $\Gamma$ with vertex set $V=\{v, w\}$ and edge set $E=\left\{e_{1}, \cdots, e_{m}, f_{1}, \cdots, f_{n}\right\}$ with $s(e)=v$ and $t(e)=w$ for all $e \in E, \Pi=\{X, Y\}$ where $X=\left\{e_{1}, \cdots, e_{m}\right\}, Y=\left\{f_{1}, \cdots, f_{n}\right\}$ for $m<n$ positive integers and $\Lambda=\Pi$. We want to figure out the nonstable $\mathcal{K}$-theory of $L:=L(\Gamma, \Pi), v L v$ and $w L w$.

The Leavitt algebra $L(m, n)$, the non-IBN algebra of type $(m, n)$ constructed by Leavitt, is isomorphic to $w L w$ [3]. Since $\mathcal{V}(L)$ is generated by $[v L],[w L]$ subject to the relations $[v L]=$ $m[w L]=n[w L]$, it follows that $[w L]$ generates $\mathcal{V}(L)$. Hence, $[w L]$ is a progenerator and $L$ is Morita equivalent to its corner algebra $w L w \cong \operatorname{End}_{R}(w L)$. In fact, $L \cong M_{n+1}(w L w)$.

Also, $\overline{\langle[v L]>}=\mathcal{V}(L)$, since $[w L] \preceq[v L]$, we get that $v L$ is also a progenerator and $v L v$ is Morita equivalent to $L$. The element $[v L v]$ of $\mathcal{V}(v L v)$ maps to $[v L]$ in $\mathcal{V}(L)$. By Corollary 16. $v L v$ is non-IBN of type $(m / d, n / d)$ where $d=\operatorname{gcd}(m, n)$. By choosing $d \neq 1$, we get examples of non-IBN Morita equivalent rings of different types.

\section{REFERENCES}

[1] G. Abrams and G. Aranda Pino, The Leavitt path algebra of a graph, J. Algebra 293(2) (2005), 319-334.

[2] G. Abrams and M. Kanuni, Cohn path algebras have invariant basis number, Comm. Alg., 44 (1) (2016), 371-380.

[3] P. Ara and K. Goodearl, Leavitt path algebras of separated graphs, J. Reine Angew. Math. 669 (2012), $165-224$.

[4] P. Ara, K. Goodearl, and E. Pardo, $K_{0}$ of purely infinite simple regular rings, K-Theory 26 (2002), 69-100.

[5] P. Ara, M.A. Moreno, and E. Pardo, Nonstable K-theory for graph algebras, Alg. Rep. Thy. 10 (2007), 157-178.

[6] G.M. Bergman Coproducts and some universal ring constructions. Trans. Amer. Math. Soc. 200 (1974), 33-88.

[7] P.A. Grillet Commutative Semigroups, Kluwer, 2001

[8] W. G. Leavitt, The module type of a ring. Trans. Amer. Math. Soc. 103 (1962), 113-130.

[9] W. G. Leavitt, The module type of homomorphic images. Duke Math. J. 32 (1965), 305-311.

[10] J. Rosenberg, Algebraic $K$-theory and its applications, GTM Springer-Verlag, 1994 
Department of Mathematics, Düzce University, Konuralp Düzce 81620 Turkey E-mail address: muge.kanuni@duzce.edu.tr

Department of Mathematics, University of Oklahoma, Norman OK U.S.A. E-mail address: mozaydin@ou.edu 